\title{
Politique
}

\section{Répertoire des thèses et mémoires en science politique,} 1985-1986

Numéro 10, automne 1986

Opinions et votes

URI : https://id.erudit.org/iderudit/040544ar

DOI : https://doi.org/10.7202/040544ar

Aller au sommaire du numéro

Éditeur(s)

Société québécoise de science politique

ISSN

0711-608X (imprimé)

1918-6584 (numérique)

Découvrir la revue

Citer ce document

(1986). Répertoire des thèses et mémoires en science politique, 1985-1986.

Politique, (10), 149-154. https://doi.org/10.7202/040544ar d'utilisation que vous pouvez consulter en ligne.

https://apropos.erudit.org/fr/usagers/politique-dutilisation/ 


\section{Répertoire des thèses et mémoires en science politique 1985-1986}

\section{Université Laval}

Bernier, Luc,

Boivin, Dominique,

Bourque, Lauri; Côté, Charles;

Daykin, Harold C.,

Diop, Cheick,

Dompierre, Daniel,

Duchesneau, Paule,

Gaboury, Hélène,

Gagnon, Michèle,

Gauthier, William,

Groleau, Gisèle,

Hachey, Jean-Marc,

Laflamme, Jacques,

Lefebvre, André,

Martin, Josée,

McKinnon, Claire,

McNamara, Marie,

Mekkaoui, Abderrahmane,

Noël, Lucie,
«Politiques gouvernementales et sociétés d'État: le cas de la caisse de dépôt et placement au Québec».

"Une nouvelle conceptualisation de la pression et du lobbying en science politique».

«Peace for the west bank: issue definition during the camp David Era with a critique of the 1983 heller report".

«La stratégie africaine des États-Unis depuis 1975. Une analyse géopolitique».

"La participation ouvrière à la gestion des entreprises en Tchécoslovaquie: genèse et limites".

"Adéquation entre l'activité électorale et l'activité législative».

"Réalignements et changements politiques au Québec: 1928-1948".

«La gestion étatique de la production humaine: la loi canadienne sur l'avortement».

"Le conflit sino-soviétique et la revision de la politique extérieure chinoise 1969-1972: les explications idéologiques fournies aux pays du Tiers-monde et aux mouvements révolutionnaires".

"Intégration des pays de l'Europe de l'Est à l'économie mondiale».

"Ghana's Political Culture of State-Economic Relations: the Asante, British, and Nkrumah Periods".

«Les industries de l'imaginaire et l'imaginaire nationaliste (critique de l'économie politique de la culture)».

"Les autorités canadiennes face au terrorisme».

"Les groupes de quartier: analyse comparative des villes de Québec et de Genève».

«Les formules de gouverne: une analyse appliquée au cas du Québec 1944-1970».

"Le 'nouveau nationalisme' au Québec: 1965 à 1976».

"L'O.U.A. et la crise saharienne: la perspective marocaine».

«Pouvoir, régulation et condition féminine au sein d'une collectivité sacrale contemporaine: le cas des témoins de Jéhovah au Québec». 
Pouliot, Jean-François,

Quenneville, Benoît, Rhéaume, Luc,

Ruel, Pierre,

Schaefer, Carmen,

St-Arnaud, Bertrand, Verrette, André,

Beauchesne, Line,

Bouchard, Pierrette,

Giroux, Guy,

Labrecque, Laurent,

Laurin, Luc,

Mahmud, Farhat,

Simard, Pierre,

Vuong, Thanh Huu, Ayotte, Sylvain,

Baillargeon, Donald,

Beaulieu, Maud,

Bérard, André,

Cisneros Alzuru, Beatriz,

Cyr, Jean-François,
"Enquête sur la condition masculine: l'impact des groupes-hommes sur tes relations sociales de sexe».

"La presse écrite locale et l'ordre du jour des affaires publiques municipales en Mauricie». "Analyse du traitement journalistique de l'information politique au Québec: le cas du projet de restructuration scolaire de 1982».

"L'apport de la politique énergétique canadienne à la réalisation de l'objectif national d'indépendance économique de la troisième option: contexte des relations canado-américaines, 1973-78».

"Influence du mouvement pacifiste sur les partis politiques en Allemagne fédérale: les Verts et le SPD».

"Le contrôle parlementaire de la société Hydro-Québec (1966-1981)».

«Le programme énergétique national (PEN): la crise économique, l'État et l'internationalisation du capital canadien».

"D'une impossible démonstration à une paradoxale positivité: le dialogue juridico-politique en matière de droits de l'homme».

«Le journal: instrument idéologique d'incitation à la militance chez la ligue communiste marxiste-léniniste canadienne».

«L'information sur l'État et le non-accès à l'information en démocratie (incidences sur l'avenir du régime)».

"Les coopératives de consommation: dimensions matérielles, culturelles, sociales et du pouvoir".

"Le sénat américain et la guerre au Vietnam».

«Pakistan's Relations with the United States and the People's Republic of China in the Ayub Khan Era: 1958-1969".

«L'entrepreneurship dans les entreprises publiques: le cas du commerce des alcools au Québec (1961-1982)».

"Un modèle cognitif et décisionnel de fabrication de stratégies de changement».

"Le Canada et le développement autocentré: le cas du Bangladesh".

"La politique d'éducation du Parti québécois et les classes populaires».

«La politique industrielle de la CEE: enjeu du pouvoir législatif et budgétaire?»

«Ellul et la métaphysique de la technique».

«La CTPD: un instrument pour l'autonomie collective? Le cas du Comité d'action pour la reconstruction du Nicaragua».

«L'évolution de la structure urbaine de Québec et de ses conditions d'insertion dans le champ d'accumulation nord-américaine. 1940-1980». 
Dugas, Normand,

Dussault, André,

Ferland, Marie-France,

Fontaine, Louise,

Forand, Claude,

Fortin, Jean-Pierre,

Frédérick, Michel,

Gaudreault, Martine,

Grenier, Yvon,

Lecours, Francine,

Massie, Michelle,

Morin, Jacques,

Nadeau, Chantal,

NToutoume Emane; Simon, Wilfrid

Rhounaim, Rabia,

Sarrazin, Martine,

Tremblay, Suzanne,

Vuillet, André,

Villeneuve, Pierre,

Tremblay, Daniel,

\section{Université de Montréal}

Delorme, Pierre,

Desoer, Frank,
«La conception des relations de travail du gouvernement du Parti québécois: essai de qualification".

«La révision constitutionnelle au Canada entre 1971 et 1980: étude d'un phénomène de polarisation".

«Les conditions de la concertation et la conférence sur les communications».

"Groupes linguistiques, conflits politiques: le cas belge».

"Problèmes urbains et participation électorale à Sainte-Foy: 1969-1977".

"Incidences de la démocratisation de l'enseignement supérieur sur l'autonomie des établissements universitaires québécois».

«La création d'une zone exempte d'armes nucléaires dans les régions arctiques».

"L'analyse de contenu et l'analyse sémiotique: Études comparatives de deux méthodes». «De l'ordre à l'ordre: la révolution cubaine».

"L'URSS et le conflit Irako-Iranien».

"Analyse stratégique de deux sondages d'opinion publique».

"La position canadienne face au Nouvel Ordre Mondial de l'Information et de la communication".

"Néo-nationalisme, problèmes sociaux et partis politiques: étude des programmes de 1970 et 1981 du Parti québécois».

"L'Europe face au nouvel ordre économique international à travers l'association CEE/ACP. Essai d'analyse critique".

"Le problème du choix technologique: cas de la révolution verte en Inde».

"Introduction à l'aspect politique de l'œuvre de Georges Brassens».

"Sheng Yige Hao. La république populaire de Chine et l'implantation d'une politique démographique en milieu rural 1972-1982».

«Sahel d'hier et d'aujourd'hui : explications dominantes et succession des dominations». "La diversification structurelle des exportations canadiennes vers la Corée du sud: un échec ".

«Le dispositif gouvernemental québécois de gestion de la vieillesse: 1970-1981».

"L'État et l'ordre urbain: politiques gouvernementales et administration publique de l'espace urbain québécois».

"Action critique et changement social au Québec: l'intelligentsia de l'après-guerre et le cas de l'Institut canadien des affaires publiques». 
Bonin, Daniel,

Ricot, Jean-Claude, Lenglaré, Didier,

Benali, Belaid,

De Roussan, Yves, .

Kounou, Michel,

Orvieto, Sylvio,

Akhavanfarshchi, Firouzeh, Lévesque, Denis,

Picard, Paulo,

Gravel, Denis,

Gravel, Gérard,

Allaire, Benoît-Guy,

Chénard, Ginette,

Grenier, Ginette,

Trudeau, Denise,

Amrani, Saad

Cattaneo, Claudia

Brousseau, François,

Lareau, Louise,

Saint-Laurent, Marie-Claude,

\section{Université d'Ottawa}

Drainville, André,
"La collégialité et la légitimité du pouvoir: une application à la monarchie française sous Philippe IV Le Bel».

"La chute du gouvernement de l'unité populaire du 11 septembre 1973 au Chili».

«Défense de l'espace audiovisuel francophone du Québec: une expérience originale à TVFQ (Télévision française du Québec)».

"Attitudes, opinions et comportements politiques en France de 1981 à 1983".

"Modèle de développement, structure sociale et stabilité politique en Arabie Saoudite». «Croissance capitaliste et dépendance économique du Cameroun de 1960 à 1983: une étude critique".

"L'émergence de la stratégie du "compromis historique» élaborée par le parti communiste italien en 1973: analyse diachronique et synchronique».

"Le statut socio-politique de la femme en Iran".

«Epistémologie, paradigme et théories de l'organisation».

"Idéologie et pratique de la Centrale des syndicats démocratiques (C.S.D.)"

«Le gouvernement du parti québécois dans ses rapports de classes: bilan critique des analyses marxistes".

«Capitaliste d'État: une étude de la société algérienne de 1965 à 1978".

"Une étude du positionnement comparé des cadres de la grande entreprise et de la fonction publique québécoise».

"Le phénomène mondial de l'innovation technologique et les politiques et rapports internationaux d'un État nation: le cas du Canada au cours des années 1970».

"Maîtrise avec travaux dirigés. La notion de contrôle au sein du gouvernement canadien. La prospective».

"L'économie mondiale: état de la question».

«Étude comparative de la politique étrangère du Maroc et de l'Arabie Saoudite de 1970 à 1980 ".

«La couverture de presse du Moyen-Orient par les média canadiens».

"Les intellectuels polonais et Solidarnosc».

"Un bilan de l'accord cadre Canada/CEE».

«Les moyens non contraignants de défense d'un État face à une menace sécessionniste: le cas canadien en 1980 ».

«Valeur morale et déterminante de la politique étrangère des États-Unis (1970-1984)». 
Baillargeon, Stéphane,

Carignan, Diane,

Delnegro, Luciano,

Fortin, Johanne,

Labelle, Gilles,

Madden, Lucie,

Marien, Daniel,

M'Beko, Maurice-Honoré,

Perron, Claude,

Rabbaj, Benachir,

Elquzoui, Abdelfettah,

Rihani, Abdellah,

Sarda, Louise,

Uribe, Victor,

Vaillancourt, Yves,

Boyer, Richard,

Charest, Suzanne,

Desrosiers, Guy,
«La notion d'idéologie chez Althusser, Ansart, Baechler et Fossaert: spécification et réduction conceptuelle».

"Le mouvement des écoles alternatives 1956-1985".

"Canada's immigration policy, immigration legislation, and immigrant labour in 1970's».

"Bilan de l'organisation du sommet québécois de la jeunesse tenu au Québec en 1983».

"La conception du politique chez Claude Lefort".

"Les relations politico-économiques de la France avec l'Amérique latine: de 1970 à nos jours».

"La politique sociale du président Reagan à travers l'analyse de ses initiatives concernant quelques programmes sociaux significatifs (1981-1984)».

"Analyse des fondements de la coopération bilatérale entre le Canada et les pays en voie de développement: le cas de la coopération avec les États francophones d'Afrique: 19611965 ».

«Évolution des négociations namibiennes, la médiation occidentale autour du groupe de contact (1977-1983)".

"Le déficit alimentaire du Maroc».

"L'État post-colonial, les transformations des rapports sociaux de production et l'accumulation: le cas de la Tanzanie».

«Délégitimation du pouvoir et mouvement de masse: la crise du 26 janvier 1978 en Tunisie».

"La sous-alimentation et la malnutrition en Afrique face à l'agriculture d'exportation". "Le dialogue nord-sud, la dette externe et le développement économique d'Amérique latine: perspectives latino-américaines".

"Monde contemporain, autonomie et révolution: rétrospective et commentaire de l'œuvre de Cornelius Castoriadis".

"L'opération 20,000 logements de la Ville de Montréal: l'analyse d'un programme et de son support organisationnel».

"Analyse de la pensée politique de Pierre Vadeboncœur".

"Structure agraire des pays occidentaux et mode de production capitaliste: rétrospective et analyse de la méthode marxiste». 
Emard, Claude,

Izzi, Michael,

Lapointe, Daniel,

Lemay, Gaétan,

Paquerot, Sylvie,

Prévost, Jean-Guy, Stock, Brigitte,

\section{Université McGill}

Magnant, Michel,

Ostrander, Gregory,

Bourque, Bernard,

Brochard, Patrick,

Da Silva, Bernadette,

De Volder, Guido,

Gondwe, Carlton,

Margolian, Michael, McCabe, Gerald,

Schull, Joseph,

Selim, Guy Rukh,

Siaroff, Alan,

Smith, Hevina,
«Les politiques d'information municipale dans les grandes villes: une illustration Montréal 1960-1985».

"Towards a Theoretical Interpretation of Gramsci's Southern Question».

«Pouvoirs des intellectuels et démocratie dans les groupes populaires».

«L'information internationale fait-elle l'objet d'un traitement distinct dans une programmation télévisée».

«Genre féminin et participation à la gestion de la société: une étude de cas: Les femmes de la haute fonction publique québécoise".

«Raymond Aron devant les crises: Essai sur l'éthique d'un intellectuel français».

«L'image de la menace soviétique à travers trois hebdomadaires français». (1979-1981).

"The Implementation of Axiomatic Method in Political Science: A Justification".

"Knowledge and Experience in the Work of Michel Foucault".

"The Language of Nuclear Deterrence".

"The Political Economy of China's Foreign Trade».

"On the Dynamic Nature of the State: The State in Uganda from the Colonial Period to $1971 »$.

"Cooperation and Conflict in Bi-ethnic of Dual Societies: The Development of FrenchCanadian and Afrikaner Nationalism".

«Dependency, Economic Integration and Development in Developing Areas: The Cases of EAC, ECOWAS and SADCC».

"Requirements for Cooperation in Strategic Arms Control».

"Regulation of the Telephone Industry in Canada: the Formative Years».

"The Relationship of the Russian Intelligentsia to the Russian Tradition of Politics".

"Political Change and the Peasantry in Bangladesh".

"The Comparative Politics of Full Employment».

«Economic Dependence and Malawi's Foreign Policy Posture Towards South Africa». 